\title{
Percepção da família quanto aos cuidados de enfermagem em uma Unidade de Terapia Intensiva Neonatal
}

\author{
The family's perception of nursing care in a Neonatal Intensive Care Unit \\ Percepción familiar de los cuidados de enfermería en una Unidad de Cuidados Intensivos \\ Neonatales
}

Recebido: 19/08/2021 | Revisado: 24/08/2021 | Aceito: 29/08/2021 | Publicado: 31/08/2021

Elizabeth Mesquita da Silva

ORCID: https://orcid.org/0000-0001-9454-8699 Centro Universitário Christus, Brasil

E-mail: elizabethmesqs@gmail.comr

Lueyna Silva Cavalcante

ORCID: https://orcid.org/0000-0003-3920-2462 Prefeitura Municipal de Fortaleza, Brasil E-mail: usilvacavalcante@gmail.com

Ingrid Martins Leite Lúcio

ORCID: https://orcid.org/0000-0002-2822-1599 Universidade Estadual de Alagoas, Brasil E-mail: ingridmll@eenf.ufal.br

Isadora Araujo Rodrigues ORCID: https://orcid.org/0000-0002-0691-3818 Centro Universitário Christus, Brasil E-mail:isadorarodrigues52@gmail.com

Alisson Salatiek Ferreira de Freitas ORCID: https://orcid.org/0000-0002-4547-5785 Universidade Estadual do Ceará, Brasil E-mail: salatiek@gmail.com

\begin{abstract}
Resumo
A Unidade de Terapia Intensiva Neonatal, é responsável por recém-nascidos de 0 a 28 dias graves ou potencialmente graves, tendo em vista que possui estrutura e equipe especializada para proporcionar o suporte de vida necessário. Para os pais este ambiente é estressante e nem sempre a equipe os prepara para cuidados com o filho. Assim, objetivou-se compreender a percepção da família diante do cuidado de enfermagem ao recém-nascido na Unidade de Terapia Intensiva Neonatal. Trata-se de um estudo de campo, com abordagem qualitativa, realizado em um hospital de atenção secundária da rede pública de saúde, foi realizada entre os meses de janeiro e junho de 2021 com dez familiares de recém-nascidos internados na unidade, por meio de entrevistas semi estruturadas e assim, conforme as falas foram delimitadas três categorias temáticas a serem analisadas. As falas demonstraram a dificuldade de uma comunicação efetiva na relação profissional-família, na falta de identificação do enfermeiro, falhas na educação em saúde e na preparação para a alta hospitalar. Portanto, conclui-se que a família tem uma boa percepção acerca dos cuidados de enfermagem, mas que ainda há condições a serem melhoradas para um cuidado amplo e humanizado.
\end{abstract}

Palavras-chave: Família; Enfermagem neonatal; Unidade de Terapia Intensiva Neonatal. Recém-nascido; Covid-19.

\begin{abstract}
The Neonatal Intensive Care Unit is responsible for newborns from 0 to 28 days that are severe or potentially serious, given that it has a specialized structure and team to provide the necessary life support. For parents, this environment is stressful and the team does not always prepare them for child care. Thus, the objective was to understand the perception of the family regarding nursing care for newborns in the Neonatal Intensive Care Unit. This is a field study, with a qualitative approach, carried out in a secondary care hospital of the public health network, carried out between January and June 2021 with ten relatives of newborns admitted to the unit, through of semi-structured interviews and so, according to the speeches, three thematic categories were delimited to be analyzed. The statements showed the difficulty of effective communication in the professional-family relationship, the lack of identification of the nurse, failures in health education and preparation for hospital discharge. Therefore, it is concluded that the family has a good perception about nursing care, but that there are still conditions to be improved for a broad and humanized care.
\end{abstract}

Keywords: Family; Neonatal nursing; Neonatal Intensive Care Unit. Newborn; Covid-19. 


\begin{abstract}
Resumen
La Unidad de Cuidados Intensivos Neonatales se encarga de los recién nacidos de 0 a 28 días que sean severos o potencialmente graves, dado que cuenta con una estructura y equipo especializado para brindar el soporte vital necesario. Para los padres, este entorno es estresante y el equipo no siempre los prepara para el cuidado infantil. Así, el objetivo fue conocer la percepción de la familia sobre el cuidado de enfermería al recién nacido en la Unidad de Cuidados Intensivos Neonatales. Se trata de un estudio de campo, con abordaje cualitativo, realizado en un hospital de atención secundaria de la red de salud pública, realizado entre enero y junio de 2021 con diez familiares de recién nacidos ingresados en la unidad, mediante entrevistas semiestructuradas y así, según los discursos, se delimitaron tres categorías temáticas para ser analizadas. Las declaraciones evidenciaron la dificultad de la comunicación efectiva en la relación profesional-familiar, la falta de identificación de la enfermera, fallas en la educación para la salud y preparación para el alta hospitalaria. Por tanto, se concluye que la familia tiene una buena percepción sobre el cuidado de enfermería, pero que aún quedan condiciones por mejorar para un cuidado amplio y humanizado.
\end{abstract}

Palabras clave: Familia; Enfermería neonatal; Unidad de Cuidado Intensivo Neonatal; Recién nacido; Covid-19.

\title{
1. Introdução
}

A Política Nacional de Humanização (PNH), define que humanizar é promover a valorização dos sujeitos inseridos no contexto de saúde, e dentre as diretrizes da PNH, o acolhimento pretende sustentar a relação entre profissional e comunidade, proporcionando uma escuta ativa, o apoio, a empatia, e dessa forma, a integralidade da assistência por meio da criação de vínculo e compromisso (Azevedo, Lançoni Júnior \& Crepaldi, 2017).

Diante disso, o HumanizaSUS, dispõe sobre práticas de humanização ao parto e nascimento, visando proporcionar ao recém-nascido e família, uma assistência holística. Os processos de gestação, parto e nascimento são momentos marcantes na vida dos pais, portanto, todos os envolvidos devem lhes proporcionar um ambiente seguro e humanizado (Brasil, 2017).

A formação de uma família ou a chegada de uma nova criança é um momento significativo na vida dos pais, tendo em vista que desde a confirmação da gravidez, a gestação e o próprio parto, são criadas expectativas diante do nascimento e primeiros momentos com a criança, que podem ser interrompidos com a internação do recém-nascido (Kegler, Neves, Silva, Jantsch, Bertoldo \& Silva, 2019)

A Unidade de Terapia Intensiva Neonatal (UTIN), é responsável pelo cuidado integral ao recém-nascido grave ou potencialmente grave, contribuindo para um cuidado especializado, com equipe e estrutura necessária (Marski, Facio, Castanheira; Ichisato, Barba \& Wernet, 2018). Durante a internação da criança nesta unidade, a assistência ao recém-nascido deve preconizar o fortalecimento de vínculo entre pais e filho, assim como o estímulo ao aleitamento materno e o acesso a cuidados especializados ao recém nascido em risco.

A comunicação de uma intercorrência e internamento da criança em uma Unidade de Terapia Intensiva, proporciona aflição, impotência e frustração para os pais e família. No Brasil, o artigo 12, da lei 8.069, dispõe que as instituições de saúde devem promover condições propícias para a permanência integral de um dos pais durante a internação da criança ou adolescente (Brasil, 1990). no entanto, por vezes a instituição ou a própria equipe dificulta a permanência dos pais e acompanhantes, deixando-os ainda mais receosos em relação aos profissionais e o cuidado recebido pela criança.

O cuidado centrado na família busca promover uma assistência humanizada que contemple além do paciente, sendo baseada em fatores como o estímulo de cuidado dos pais durante o internamento, a valorização e respeito quanto a seus valores e crenças como a informação compartilhada, no entanto, a equipe de enfermagem apesar de saber a importância da família e tentar proporcionar um ambiente acolhedor, não compreende o significado do cuidado centrado na família e por vezes sequer conhecem o modelo de assistência (Rodrigues, Uema, Rissi, Felipin \& Higarashi, 2019)

Tendo em vista que a enfermagem é uma profissão que visa a qualidade de vida da pessoa, família e comunidade, é de suma importância que o cuidado de enfermagem contemple não apenas o paciente, mas também seus familiares e todo o contexto em que está inserido. Portanto, a equipe deve tentar minimizar a dor envolvida nesse processo através de uma comunicação clara entre profissionais e família, promovendo o cuidado integral e humanizado (Lima \& Mazza, 2019). Para mais, o presente estudo 
pretende colaborar com os profissionais a ampliar a percepção sobre o quanto a família também necessita de cuidados tanto quanto o paciente em questão.

Dessa forma, o interesse pela pesquisa surgiu durante o estágio extracurricular em uma Unidade de Terapia Intensiva Neonatal, onde emergiu o questionamento: Qual a percepção da família sobre o cuidado de enfermagem ao recém nascido em uma UTI Neonatal? Assim, objetiva-se compreender a percepção da família diante do cuidado de enfermagem ao recém-nascido na Unidade de Terapia Intensiva Neonatal.

\section{Metodologia}

Trata-se de um estudo de campo, descritivo, de caráter exploratório, transversal com abordagem qualitativa. O estudo qualitativo consiste no ato de compreender aspectos singulares e subjetivos dos participantes (Minayo, Diniz \& Gomes, 2016).

O estudo foi realizado em um hospital de atenção secundária da rede pública de saúde, com o título de acreditação hospitalar nível III, excelência, pela Organização Nacional de Acreditação (ONA), localizado em Fortaleza-CE, Brasil. Trata-se de um hospital de apoio para a rede terciária de assistência do Estado do Ceará, atendendo exclusivamente a clientela do Sistema Único de Saúde (SUS). O setor escolhido foi a Unidade Neonatal de Alto risco, composta de 8 leitos na Unidade de Terapia Intensiva de Neonatologia.

A população de estudo foi composta por familiares de recém-nascidos internados na unidade, independente do tempo ou motivo de internação do RN. Todavia, devido a pandemia no Novo Coronavírus, os hospitais reduziram o tempo de visitas e os acompanhantes não eram permitidos para diminuir a circulação de pessoas dentro dos hospitais visando a diminuição do contágio da doença, dificultando assim a inclusão de participantes e consequentemente, a quantidade de amostra da pesquisa.

A abordagem aos participantes foi na Unidade Neonatal, os quais foram convidados a participar da pesquisa, e assim, conduzidos a uma sala reservada para a entrevista. O total de participantes foi definido por critério de saturação, sendo definida pela suspensão das entrevistas quando o pesquisador considera que há redundância ou repetição nos dados coletados (Fontanella, Ricas \& Turato, 2008)

A coleta de dados ocorreu no período de janeiro a maio de 2021 por meio de um roteiro de entrevista semi estruturada, assim como áudio-gravadas e compostas por questões que identifiquem o perfil, e caracterizem os principais questionamentos do estudo buscando responder aos objetivos da pesquisa.

Deste modo, os dados obtidos foram analisados por meio da caracterização dos dados, cujos resultados foram transcritos e verificados a partir da saturação das falas (Minayo et. al 2016) de maneira que surgiram as seguintes categorias temáticas: Acolher e Interagir: Ações necessárias para o cuidado; O ser enfermeiro e sua assistência sob a perspectiva dos pais; A alta como um desafio para o cuidado.

Ademais, o presente estudo seguiu as normas da Resolução no 466/12 do Conselho Nacional de Saúde do Brasil, responsável pelos princípios éticos e científicos fundamentais de pesquisas com seres humanos (Brasil, 2013) assim, o estudo foi aprovado pelo Comitê de Ética sob parecer 4.730.552.

\section{Resultados e Discussão}

Foram entrevistados 10 familiares, destes, sete eram mães e três pais. A idade variou entre 19 e 44 anos de idade, seis possuem o ensino médio completo e quatro, o ensino fundamental. Em relação à quantidade de filhos, oito dos entrevistados relataram que o RN era seu primeiro filho, enquanto dois dos participantes disseram ser seu terceiro filho.

Desta forma, quanto às condições de pré-natal e nascimento do recém-nascido, nove das mães realizaram consultas prénatal e uma não, dentre as nove, cinco tiveram menos de seis consultas e quatro tiveram mais de seis. Em relação ao parto, seis 
deles foram vaginais e quatro partos cesarianos.

Ademais, o peso dos recém nascidos variou entre $800 \mathrm{~g}$ e $3.555 \mathrm{~g}$, em relação ao comprimento obteve-se valores entre 33 e $50 \mathrm{~cm}$. Sete dos neonatos estavam internados devido a prematuridade ou ganho de peso, enquanto um por crise convulsiva e dois por alterações cardiopulmonares.

De acordo com a Organização Mundial da Saúde (WHO, 2018) o recém nascido considerado prematuro é definido por nascidos antes de 37 semanas de gestação, sendo ainda dividido entre prematuro extremo quando nascidos com menos de 28 semanas gestacionais, prematuro moderado quando nascidos entre 28 e 34 semanas e o prematuro tardio quando nascidos entre 34 e 37 semanas. O nascimento de uma criança pré-termo ocorre por motivos multifatoriais, e as complicações do parto prematuro são as principais causas de mortalidade em menores de cinco anos.

A comparação entre a quantidade de consultas pré-natal e a prematuridade dos neonatos, é até duas vezes maior em comparação às gestantes que realizam a quantidade mínima de consultas e que iniciam o pré natal antes da $16^{\circ}$ semana de gestação. Assim, quatro dos sete recém nascidos que nasceram prematuros, foram realizadas menos de seis consultas de acompanhamento neonatal, evidenciando assim, a importância do pré natal no cuidado e prevenção de intercorrências à gestante e recém-nascido (Melo, Oliveira \& Mathias, 2015).

\section{Acolher e interagir: ações necessárias para o cuidado}

A presença dos pais na Unidade de Terapia Intensiva Neonatal é considerada um cuidado holístico onde tem-se a abrangência do paciente e família, entretanto indica maiores responsabilidades da equipe em relação aos familiares, tendo em vista que estes por vezes não compreendem os procedimentos ou rotina da unidade. Vale ressaltar que é necessária uma comunicação efetiva e bilateral, e esta, deve começar desde o diagnóstico e se prolongar por toda a permanência da criança no ambiente hospitalar.

Quando questionados a respeito dos motivos de permanência e /ou internações, têm-se as seguintes percepções:

E4: "Por conta dele ser prematuro né, está se alimentando pela sonda, ai tão fazendo essa adaptação por que ele só pode sair ou mamando ou na mamadeira"

E8: "Por causa da regurgitação, ele tava voltando o leite..."

E9: "É por causa que ela deu muito trabalho ao nascer, forçaram muito a barriga no hospital...e ela comeu muito, minha esposa, o médico disse que não era pra comer nada mas aí ela comeu escondido, aí por isso a criança nasceu desse jeito, sem conseguir engolir nada..."

Pode-se observar conforme as falas anteriores o quanto a convivência na unidade promove uma familiarização dos pais com relação à condição de saúde do neonato e a termos técnicos até então desconhecidos por eles tornam-se normais e estes sentem-se mais próximos da equipe e do cuidado com seu filho.

No entanto, ao se comparar o motivo de internação e/ou permanência contida no prontuário do recém nascido com o relato dos pais, percebe-se uma divergência de informações, demonstrando o quanto ainda há falhas de comunicação entre a equipe e a família, seja da instituição anterior ou após a transferência à UTIN. Percebemos também o quanto a compreensão da doença pode estar relacionada a uma bagagem cultural, por vezes, baseada em crenças populares.

Em um estudo, buscou-se diferenciar a informação sobre o estado de saúde do paciente com a comunicação, sendo o primeiro um breve relato sobre o quadro clínico enquanto a comunicação perpassa a orientação, exigindo um envolvimento e vínculo maior entre o comunicador e o comunicado (Campos, Silva, Bernardes, Soares \& Ferreira, 2017). 
Deste modo, cabe a equipe estabelecer uma boa comunicação com os familiares desde o primeiro momento na unidade, gerando acolhimento e vínculo, os quais promovem a sensação de segurança e confiança no cuidado, como pode se observar nas falas a seguir:

E2: "É o que me deixa tranquila a ir pra casa, porque a gente não pode dormir aqui né? É isso, saber que aqui ela está sendo bem cuidada mesmo..."

E3: "Sim, é ótimo aqui, eu vejo como elas têm cuidado[...] a enfermagem sempre é boa, é porque eu vejo o cuidado delas... estão sempre ali..."

A visitação aberta dos pais proporciona um vínculo que os tranquiliza, uma vez que ao acompanhar os procedimentos realizados, entender o funcionamento da unidade e assim, comprovar a qualidade do cuidado, é possível criar confiança e segurança em relação à equipe (Banhara, Farinha, Henrique, Razera, Alves \& Trettene, 2018)

Todavia, a presença dos pais por vezes pode causar desconforto tanto nos profissionais quanto aos familiares, uma vez que, quando estes não são bem informados sobre a necessidade de um procedimento invasivo, quando não há uma comunicação mais empática ou uma escuta ativa que demonstre compreensão e apoio, tendem a ver uma mecanização nos cuidados ou não entendem a necessidade de um procedimento que apesar de causar dor, é indispensável para o tratamento de seu filho, como observado a seguir:

E7: "São boas, cuidam super bem...é só que pelo costume de lidar com isso eu acho que pra eles é algo comum, mas eles cuidam muito bem [..] às vezes eu acho que falta um pouco mais de humanidade, elas cuidam muito bem, fazem o trabalho direito, mas é que eu acho que isso acaba ficando tudo normal né? pelo costume deles de lidar com isso todo dia...e no caso pra gente tá vivendo isso pela primeira vez, é algo novo, é mais dificil e complicado"

As falas demonstram o quanto a família ainda carece da atenção e do suporte da equipe, pois apesar de considerar o cuidado adequado, tem-se uma visão mecanicista e por vezes insensível da equipe em relação aos procedimentos, devido a dor que possa estar causando ao recém-nascido. É sabido que por vezes alguns procedimentos são necessários para a assistência de saúde, entretanto quando não se tem noção da importância ou necessidade, só é possível pensar na dor, portanto, explicar e demonstrar apoio e entendimento sob a dor dos pais é essencial para promover a humanização em uma unidade neonatal.

A humanização é compreendida, como uma assistência que se desenvolve na relação profissional-família, o acolhimento como sua principal diretriz, onde o profissional deve se responsabilizar por este ser desde sua chegada até sua saída, fazendo uso principalmente de uma escuta qualificada (Martins, Azevedo \& Afonso, 2018).

Tais cuidados, no entanto, devem ser igualitários à mães e pais, considerando que ambos têm direito de participar da evolução de seu filho tendo em vista que isto proporciona um vínculo à família que acabou de se formar. Todavia, observa-se uma fragilidade nos cuidados quando estes condizem aos pais das crianças.

Quando questionado sobre as informações da saúde do RN e se foram estimulados a cuidar do neonato durante as visitas, alguns dos pais responderam:

E6: "Sim, estão ligando [...] não sei dizer quem é... geralmente falam com minha esposa"

E10: " não, geralmente falavam com a avó...quando vim agora pra cá eles me falaram como ele está [...] Cuidar não... quando eu venho eles me falaram assim como ele tá..." 
A importância dos pais no cuidado e acompanhamento do RN em uma UTIN, deve ser encarado como algo que vai para além de ser um suporte para a mãe, é parte integrante deste núcleo familiar e para a criança reforça o laço paterno possibilitando assim, experiências proprioceptivas, cognitivas, perceptivas além da afetiva (Santos \& Guarany, 2019)

Portanto, tanto quanto as mães, os pais devem receber informações sobre a evolução de seu filho, ser convidado e estimulado a cuidar do neonato, incentivando o laço afetivo entre o recém-nascido e família, tal vínculo está também relacionado ao tempo em que os pais conseguem ir a unidade e acompanhar o processo de internação, o que pode ser dificultado por algumas situações como residir em outra cidade ou ter mais de um filho para cuidar, entretanto no cenário de pandemia, têm-se além dessas, a restrição da circulação de pessoas nos hospitais:

E1: To achando meio ruim só essa coisa de entrar de três horas sabe, porque aqui dizem que é acompanhante, mas lá embaixo me dizem que só posso entrar três horas da tarde, aí eu desmamo né e vou entrar só esse horário? [...] Eu venho agora de manhã e aí passo o dia, mas ontem me barraram aí eu me alterei, e hoje de novo, porque se é acompanhante e eu quero acompanhar cada minutinho dela. Aí eu fico lá direto, elas deixam eu dar o leite pra ela pela sonda, segurar ela..."

As restrições das famílias apenas a uma breve visitação, dificulta o fortalecimento de vínculo entre a família e o recémnascido como também da realização do cuidado centrado na família tendo em vista que estes não estavam acompanhando a internação. Ademais, devido o cenário de pandemia, além das habituais angústias com relação a saúde de seu filho, há também o receio de não poder estar acompanhando os cuidados, o medo de contaminação cruzada entre equipe-pacientes e da possibilidade de contaminar o próprio filho:

E1: "Eu to achando ruim assim né porque aqui é do lado da covid aí tenho medo dela pegar ou os profissionais passarem pra ela, não sei, mas acho que na minha relação com eles não..."

E2: "Só medo mesmo, porque aqui fica perto da covid ne? Aí tem muitas crianças prematuras e eu tenho medo dela ficar doente..."

E4: "Ela atrapalha porque assim, a gente tem medo de vir aqui e trazer né, porque é às vezes uma doença silenciosa e você pode ta assintomática e trazer, assim a gente fica com medo e com certeza atrapalha demais, até porque aonde eu tava eu não consegui ver ele por vinte dias, porque lá não estava tendo visita aí passei a ter depois que vim pra cá"

Evidencia-se o quanto uma internação durante uma pandemia pode proporcionar ainda mais aflições com relação ao estado de saúde do paciente, considerando que este está sob risco de vida devido a uma intercorrência e que esta pode se agravar com a contaminação de outra doença (Waddington, Van Veenendaal, O'Brien \& Patel, 2021). Para mais, há receio dos pais com relação a interagir com o neonato por medo de the contaminar e piorar seu estado de saúde, assim, novamente vê-se a necessidade da equipe de informar os familiares quanto às precauções antes e após o contato com o recém-nascido assim como as medidas de precaução que a equipe está utilizando para que assim, possa os tranquilizá-los em relação aos riscos.

\section{O ser enfermeiro e sua assistência sob a perspectiva dos pais}

A enfermagem é a maior categoria da área da saúde, sendo indispensável para o bom funcionamento da assistência, é composta por auxiliares de enfermagem, técnicos e enfermeiro (Amorim, Souza, Pires, Ferreira, Souza \& Vonk, 2017). Os 
cuidados de enfermagem são contínuos, deste modo, estão sempre presentes durante a internação do paciente, sendo diariamente observados pelos usuários seja por uma boa ou má perspectiva. Assim, quando questionados sobre os cuidados da equipe de enfermagem obteve-se as seguintes falas:

E3: "Sim, é ótimo aqui, eu vejo como elas têm cuidado [...] a enfermagem sempre é boa, é porque eu vejo o cuidado delas... estão sempre ali..."

E4: "Eu gostei muito da forma que eles tratam as crianças até mesmo nós...eles são bem atenciosos, tudo que a gente pergunta eles respondem...gostei muito”

E8: "Vai variando de enfermeira, tem umas que cuidam muito bem, mas tem outras que não ligam pra nada ... só dáo banho, alguma coisa e coloca ele lá, aí o resto fica com a gente..."

Diante das falas, pode-se observar o quanto a qualidade do cuidado é associada ao tempo em que os pais tem disponível para acompanhar seus filhos como também ao "estar sempre ali" da equipe de enfermagem, logo, a assistência de qualidade é vista como o estar realmente disponível para o cuidado, seja ser presente para a criança ou mesmo para ouvir e sanar dúvidas dos pais.

Os familiares percebem os cuidados e conseguem distinguir quem eles consideram um bom ou ruim profissional. Para os pais, o cuidado ao RN baseado na humanização, está diretamente interligado a atenção destinada à criança e ao vínculo da família criado com a equipe em questão (Noda, Maria Alves, Gonçalves, Silva, Fusco \& Avila, 2018)

Por vezes, os usuários confundem os profissionais e acreditam que todos os integrantes da equipe de enfermagem são de fato um enfermeiro do setor, ou mesmo não sabem dizer quem os informou ou foi responsável por algum cuidado e/ou procedimento que tenha sido executado. Como se pode constatar nas falas a seguir:

\section{E1: "Todo dia é uma enfermeira, acho que é enfermeira, não me lembro o nome dela..." \\ E2: "Sim, todo dia, eu acho que é uma médica..."}

Desta forma, as falas demonstram que os profissionais não costumam apresentar-se, dificultando aos usuários diferenciar quem são. Os usuários ainda possuem dificuldade na diferenciação dos papéis da equipe de enfermagem, tendo em vista que estes não se apresentam ao realizar o cuidado e, por vezes, confundem suas próprias atribuições dentro da equipe ou com outros profissionais da saúde, colaborando ainda mais para a falta de representatividade e reconhecimento do ser enfermeiro (Amorim, Souza, Pires, Ferreira, Souza \& Vonk, 2017).

\section{A alta como um desafio para o cuidado}

A tão esperada alta hospitalar, para os pais, é finalmente poderem ter seu filho em casa como sempre idealizaram. E assim, este não está mais em perigo de vida e será cuidado em casa com a presença constante da família, entretanto, para alguns pais pode gerar um sentimento de receio, tendo em vista que o recém-nascido passou há pouco tempo por uma intercorrência que o deixou sob cuidados intensivos e que agora estarão sob sua responsabilidade. Quando interrogados sobre sua segurança em cuidar do recém-nascido após a alta têm-se as falas: 
E1: "Porque ela é pequena né? mas como eu tenho duas, cada dia é uma etapazinha, dela eu não sei, é um pouco de medo de segurar...!"

E3: "Pra mim cuidar dela ainda não, tenho receio, ainda não recebi orientação, assim só de mãe, irmã que já tiveram filhos já, mas aqui só sobre amamentação até agora...”

E4: "Sim, aqui eles falam né, e sempre tem pessoas que ficam falando, aqui conheci algumas mãezinhas que já foram mães e orientam também"

Percebe-se mediante as falas, que estes que ainda há certa dificuldade dos usuários em recorrer aos profissionais quando há dúvidas, e então suprem essa falta através da troca de experiências entre mães e pais que apesar de ser uma vivência válida, pode ser ou não benéfica para a criança tendo em vista a falta de respaldo científico.

Os pais diversas vezes recebem alta hospitalar com dúvidas sobre de atividades básicas, tais quais alimentação, higiene e desenvolvimento do recém-nascido, e que estas não são retiradas na UTIN por estresse devido a condição de saúde do neonato ou por vergonha em relação aos profissionais, sendo assim, é preferível que estas sejam repassadas através de educação em saúde diariamente durante a internação do neonato (Silva, Fujinaga, Brek \& Valenga, 2021).

Ao questionados em relação às orientações sobre os cuidados com o recém nascidos têm-se outras falas:

E1: "Não ainda não, mas acho que porque ela ainda vai pro berçário ne..."

E7:" Sim, sei os cuidados, ainda não fui orientada, mas acho que quando tiver mais perto vou ter mais, me falaram um pouco sobre alimentação e banho..."

Observa-se que as orientações ainda são prioritariamente recebidas apenas quando há alta hospitalar ou quando o neonato está em uma unidade com menor risco, entretanto, ao serem informados apenas durante a alta, os pais se vêem com excesso de informação que não conseguem absorver devido a emoção de finalmente levar seu filho para casa e por consequência, sentem-se inseguros em cuidar do recém-nascido.

Deste modo, conforme as categorias analisadas, é possível observar o quanto a família percebe o cuidado de enfermagem em relação ao recém-nascido, mas que para garantir um cuidado humanizado, ainda carece de assistência à família. Desde um acolhimento efetivo assegurando a valorização e respeito à sua dor, promover comunicação clara e identificação da equipe e a preparação para alta hospitalar que lhes proporcione segurança desde os primeiros momentos de internação.

\section{Considerações Finais}

Diante da percepção dos pais, a equipe de enfermagem realiza um cuidado de qualidade ao recém-nascido mas é notório que ainda há falhas em relação ao cuidado centrado na família, e que existem barreiras a serem rompidas em busca de um cuidado amplo que possa abranger a família e recém-nascido, tais quais a comunicação, a participação dos pais no cuidado e a educação em saúde contínua visando a alta hospitalar.

Para tanto, há necessidade de uma escuta ativa e uma maior sensibilidade da equipe em relação a família para que esta sinta-se também cuidada e que possa entender a equipe como um elo de confiança em relação a saúde de seu filho.

Em busca de um cuidado contínuo, a equipe deve proporcionar além de informações sobre a saúde do RN, orientações e momentos de educação em saúde como uma roda de conversa com os pais ou mesmo atividades para que estes sintam-se confortáveis em tirar dúvidas e aprender a cuidar de seus filhos desde a internação, como também estabelece vínculo e segurança 
em relação aos profissionais.

Ademais, é perceptível o quanto o cenário de pandemia atrapalhou no cuidado aos pais, considerando que estes não puderam conviver com a equipe ou estar tão presente junto ao recém nascido como gostariam, comprometendo à assistência centralizada na família assim como causando-lhes ainda mais aflições com a internação do neonato.

O estudo possui como limitação uma pequena amostra e o fato de ter sido realizado em apenas uma unidade de terapia intensiva, entretanto, acredita-se que possa gerar uma reflexão aos enfermeiros sobre a percepção dos pais em relação a seus cuidados, ao processo de ser enfermeiro e empoderar-se disso como também em relação a humanização, que por mais que seja um conceito claro, é algo subjetivo, e consequentemente deve ser individualizado para que então possa ser reconhecido.

Em vista de possíveis futuros estudos, acredita-se que sejam relevantes os que possam levar a reflexão dos próprios enfermeiros sobre como lidam com a família na unidade de internação neonatal tendo em vista a reflexão dos profissionais sobre sua prática, assim como sobre o cuidado para a alta durante o convívio na unidade faz diferença para segurança dos pais no cuidado em casa.

\section{Referências}

Azevêdo, A. V. D. S., Lançoni Júnior, A. C., \& Crepaldi, M. A. (2017). Interação equipe de enfermagem, família, e criança hospitalizada: revisão integrativa. Ciênc. Saúde Colet, 3653-3666.

Brasil, Ministério da Saúde. (2017) Atenção humanizada ao recém-nascido: Método Canguru: manual técnico 3.

Kegler, J. J., Neves, E. T., Silva, A. M. D., Jantsch, L. B., Bertoldo, C. D. S., \& Silva, J. H. D. (2019). Estresse em pais de recém-nascidos em uma Unidade de Terapia Intensiva Neonatala. Escola Anna Nery, 23.

Marski, B. D. S. L., Facio, B. C., Ichisato, S. M. T., Barba, P. C. D. S. D., \& Wernet, M. (2018). Cuidado Desenvolvimental: assistência de enfermeiros de Unidade de Terapia Intensiva Neonatal. Revista Brasileira de Enfermagem, 71, 2758-2766.

Brasil. Diário Oficial [da] República Federativa do Brasil, lei 8.069 (1990). Dispõe sobre o Estatuto da Criança e do Adolescente e dá outras providências.

Rodrigues, B. C., Uema, R. T. B., Rissi, G. P., Felipin, L. C. S., \& Higarashi, I. H. (2019). Family centered care and practice in the neonatal intensive care unit. Rev Rene, (20), 18.

Lima, V. F. D., \& Mazza, V. D. A. (2019). Necessidades de informações das famílias sobre saúde/doença dos prematuros em unidade de terapia intensiva neonatal. Texto \& Contexto-Enfermagem, 28.

Minayo, M. C. D. S., Diniz, D., \& Gomes, R. (2016). O artigo qualitativo em foco. Ciência \& Saúde Coletiva, 21(8), $2326-2326$.

Fontanella, B. J. B., Ricas, J., \& Turato, E. R. (2008). Amostragem por saturação em pesquisas qualitativas em saúde: contribuições teóricas. Cadernos de saúde pública, 24, 17-27.

Brasil, Conselho Nacional de Saúde. Resolução n 466 (2012). Aprova normas regulamentadoras de pesquisas envolvendo seres humanos. Diário Oficial da União [Internet].

World Health Organization (2018). Preterm birth. http://www.who.int/mediacentre/factsheets/fs363/en/» http://www.who.int/mediacentre/factsheets/fs363/en/.

Melo, E. C., Oliveira, R. R. D., \& Mathias, T. A. D. F. (2015). Factors associated with the quality of prenatal care: an approach to premature birth. Revista da Escola de Enfermagem da USP, 49, 0540-0549.

Campos, C. A. C. A. D., Silva, L. B. D., Bernardes, J. D. S., Soares, A. L. C., \& Ferreira, S. M. S. (2017). Desafios da comunicação em Unidade de Terapia Intensiva Neonatal para profissionais e usuários. Saúde em Debate, 41, 165-174.

Banhara, F. L., Farinha, F. T., Henrique, T., Razera, A. P. R., Alves, N. G. A., \& dos Santos Trettene, A. (2018). Visitação aberta em unidade de terapia intensiva neonatal: percepções da equipe de enfermagem [Open visitation in a neonatal intensive care unit: nursing team's perceptions][Visitación abierta en unidad de terapia intensiva neonatal: percepciones del equipo de enfermería]. Revista Enfermagem UERJ, 26, 33461.

Martins, P. L., Azevedo, C. D. S., \& Afonso, S. B. C. (2018). O papel da família nos planos de tratamento e no cuidado pediátrico hospitalar em condições crônicas complexas de saúde. Saúde e Sociedade, 27, 1218-1229.

dos Santos, R. P., \& Guarany, N. R. A experiência do pai na unidade de tratamento intensivo neonatal/The experience of the father in the Neonatal Intensive Care Unit. Revista Interinstitucional Brasileira de Terapia Ocupacional-REVISBRATO, 3(2), 230-246.

Waddington, C., van Veenendaal, N. R, O'Brien, K. \& Patel, N. (2021). International Steering Committee for Family Integrated Care. Family integrated care: Supporting parents as primary caregivers in the neonatal intensive care unit. Pediatric Investigation, 5(2), $148-154$. 
Research, Society and Development, v. 10, n. 11, e262101119597, 2021

(CC BY 4.0) | ISSN 2525-3409 | DOI: http://dx.doi.org/10.33448/rsd-v10i11.19597

de Azevedo Amorim, L. K., de Oliveira Souza, N. V. D., da Silva Pires, A., Ferreira, E. S., de Souza, M. B. \& Vonk, A. C. R. P. (2017). O trabalho do enfermeiro: reconhecimento e valorização profissional na visão do usuário. Revista de Enfermagem UFPE on line, 11(5), 1918-1925.

Noda, L. M., Alves, M. V. M. F. F., Gonçalves, M. F., Silva, F. S. D., Fusco, S. D. F. B., \& Avila, M. A. G. D. (2018). A humanização em unidade de terapia intensiva neonatal sob a ótica dos pais. Revista mineira de Enfermagem, 22.

da Silva, C. G., Fujinaga, C. I., Brek, E. F., \& Valenga, F. (2021). Cuidados com o recém-nascido prematuro após a alta hospitalar: investigação das demandas familiares. Saúde e Pesquisa, 14(2). 